\title{
Surface Preconditioning Strongly Influences the Antifouling Capabilities of Zwitterionic and Nonionic Polymer Brushes
}

Ivana Víšová ${ }^{1}$, Markéta Vrabcová ${ }^{1}$, Michala Forinová $^{1}$, Yulia Zhigunová1, Vasilii Mironovi ${ }^{1}$ Milan Houska ${ }^{1}$, Eva Bittrich ${ }^{2}$, Klaus-Jochen Eichhorn ${ }^{2}$, Hisham Hashim ${ }^{3,4}$, Petr Schovánek ${ }^{1,5}$, Alexandr Dejneka ${ }^{1}$, Hana Vaisocherová - Lísalová ${ }^{1}$

${ }^{1}$ FZU - Institute of Physics of the Czech Academy of Sciences, Na Slovance 1, Prague, Czech Republic.

${ }^{2}$ Leibniz-Institut für Polymerforschung Dresden e.V., Hohe Str. 6, 01069 Dresden, Germany.

${ }^{3}$ National University of Science and Technology (MISIS), Leninskiy prospekt 2, Moscow, 119049, Russia.

${ }^{4}$ Tanta University, Faculty of science, Al-Geish street, Tanta, 31527, Egypt.

${ }^{5}$ Palacký University Olomouc, 17. listopadu 12, 77146 Olomouc, Czech Republic.

Corresponding Author: Hana Vaisocherová-Lísalová, Ph.D., Institute of Physics, Academy of Sciences of the Czech Republic, v.v.i., Na Slovance 1, 18221 Prague, Czech Republic; Tel.: +420 2660529 93, E-mail: lisalova@fzu.cz,

Key words: surface modification, zwitterionic, antifouling functionalizable polymer brush, resistance to fouling, spectroscopic ellipsometry, surface probing

\section{Supporting Information}

\section{Polymerization procedure}

Slides of BK7 glass chips with 50nm gold layer or Au-coated QCM crystals were sonicated in pure isopropanol for $30 \mathrm{~min}$, rinsed with isopropanol, ethanol, and then immersed into a $1 \mathrm{mM}$ solution of $\omega$-mercaptoundecyl bromoisobutyrate in ethanol, which was stored for 3 days in the dark at room temperature. After, chips or crystals were rinsed with ethanol, dried, and mounted into a custom-made polymerization system developed at the Institute of Physics CAS.

For the polymerization procedure, batches of both methanol and water were first degassed using six freeze-pump-thaw cycles. The catalysts $\mathrm{CuCl}(20 \mathrm{mg}), \mathrm{CuCl} 2(6 \mathrm{mg})$, and the ligand $\mathrm{Me}_{4} \mathrm{Cyclam}(69.1 \mathrm{mg}$ ) were placed into a Schlenk tube and dissolved in $1.4 \mathrm{ml}$ of degassed methanol under a nitrogen atmosphere. The catalyst solution was then sonicated for 5 min. Monomers CBAA (2.56 g), CBMAA (2.72 g), HPMAA (1.61 g), or SBMAA (3.28 g) were dissolved in $14.5 \mathrm{ml}$ of degassed water and stirred until dissolution was completed. Both solutions (catalyst + monomer) were then mixed under nitrogen with the use of a gastight syringe. The SAM coated chips were then transferred into a reactor, after which the catalyst/monomer solution was added. The polymerization reaction was performed for $2 \mathrm{~h}$ at room temperature, 
after which the polymer brush substrates were rinsed with ultra-pure water. All substrates were then immersed in phosphate-buffered saline (PBS, $0.01 \mathrm{M}$ phosphate, $0.138 \mathrm{M}$ sodium chloride, $0.0027 \mathrm{M}$ potassium chloride) and kept at $4{ }^{\circ} \mathrm{C}$ until use.

\section{Spectroscopic ellipsometry, QCM, and SPR measurements}

Measurements in different ionic strength environments by spectroscopic ellipsometry and QCM: Polymer-coated substrates were rinsed with water properly and put into the environment of interest (buffer with specified salts added) to stabilize to room temperature and to adapt to a defined ionic strength for $30 \mathrm{~min}$. After that, they were removed and put into the measuring cuvette filled with the same degassed buffer. After a few minutes of system stabilization, ellipsometric data were collected.

QCM crystals coated with polymer brushes were washed with buffer without added salts. Then, buffer with salts was flowing into the microfluidic system for $10 \mathrm{~min}$. The decrease of the frequency of the crystal between the point when the surface was washed in buffer without added salts and after salt introduction was evaluated.

Surface rehydration measurements via ellipsometry: Polymer coated substrates stored in PBS were rinsed properly with water and kept in ultrapure water for 1 hour. The chips were then attached to the ellipsometry sample holder (Figure S1), the cuvette was filled with water, and after system equilibration, ellipsometric data were collected. Chips were then completely dried by a stream of nitrogen, left under air for 1 hour, after which spectra of dried chips were taken. For rehydration measurements, water was again added to the cuvette and left for another hour to rehydrate, after which spectra data were collected.

SPR fouling measurements: Polymer coated chips coated were thoroughly rinsed with water. To measure fouling on rehydrated coatings, chips were dried by a flow of nitrogen kept dry under air for 1 hour, after which they were mounted onto the SPR system. To measure fouling on non-dried coatings, chips were mounted onto the SPR system right after washing with water. For measurements, all chips were washed with HEPES buffer (without added salts) for $30 \mathrm{~min}$ under a flow of $30 \mu \mathrm{l} / \mathrm{min}$ to create a baseline. Then, undiluted blood plasma was introduced for $10 \mathrm{~min}$ at the same flow rate, followed by buffer (without added salts) to create an after-fouling baseline. Fouling levels were determined from the difference between the baseline level $10 \mathrm{~min}$ after undiluted blood plasma injection and the baseline before blood plasma introduction.

\section{Ellipsometric sample holder}

We designed a custom cell to meet the high demands of accurate ellipsometry in liquid environments. Specifically, we designed and fabricated an optical cuvette (BK7) that can be sealed and secured to the ellipsometer in a reliable fashion (Figure S1). This setup enables precise sample alignment, ensures data collecting from the same spot of the polymer brush samples across all different solutions, and minimizes errors caused by increasing of salt ions concentration in the solution due to evaporation during the measuring. 

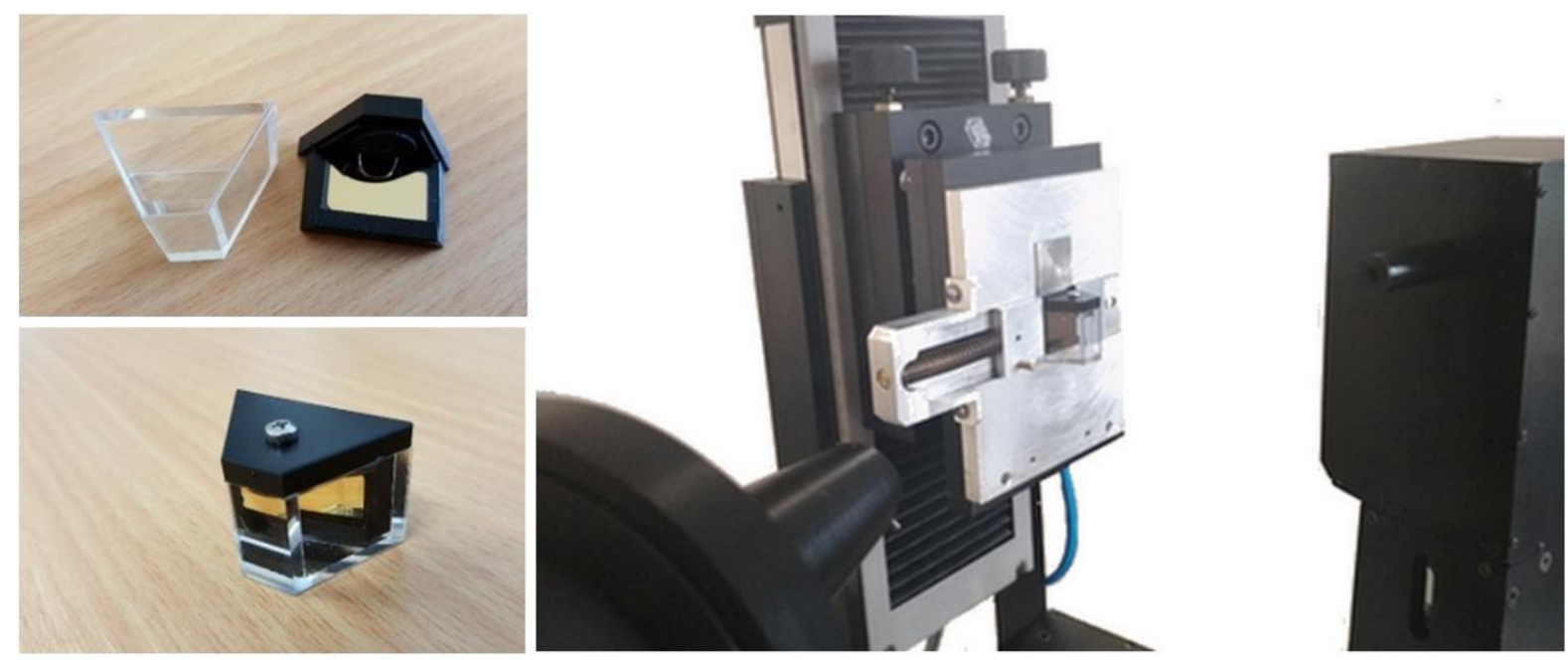

Figure S1: Custom-made cell (left), and use in ellipsometric measurements (right).

\section{Dispersion function of used liquids}

Dispersion functions of all water-based buffer solutions were measured by multi-wavelength refractometry (DSR- $\lambda$, Schmidt + Haensch GmbH \& Co., Berlin, Germany) in a range of wavelengths between $435-707 \mathrm{~nm}$. After, the functions were extrapolated in the full region of $350-800 \mathrm{~nm}$, taking into account the standard dispersion function of water. Resulting spectra describe reliably the behaviour of water-based buffers in all region $350-800 \mathrm{~nm}$.

Even though the refractive index of the high salt concentration environment appears to be close to the refractive index of polymer brush itself, the thickness uniqueness fit allows us to conclude that the calculated parameters of polymer brush structures in such environment are reliable.
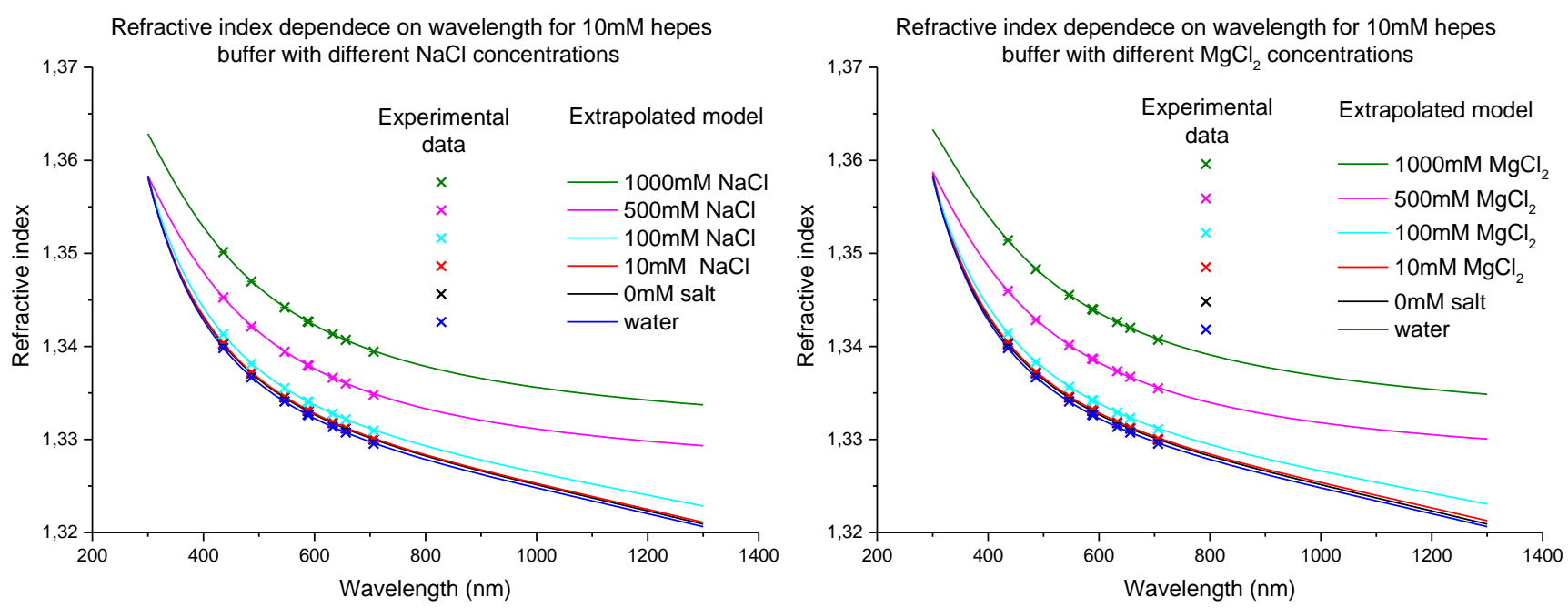

Figure S2: Refractive index spectra for all used environments. 


\section{Examples of measured and fitted ellipsometric data of polymers}

Figure S3 presents representative ellipsometric data (measured in water) for all 4 brush types used herein. The thickness range of polymer brushes was between 70 and $130 \mathrm{~nm}$, where we used different single or multi-oscillator models (Gaussian or Tauc-Lorentz oscillators).

(a) Oscillators: 1x Tauc-Lorentz (amplitude $6.60 \pm 1.22$, Eg $3.08 \pm 0.09$, C $8.72 \pm 3.73$ ), MSE 0.96.

pCBAA in water, $70^{\circ}$

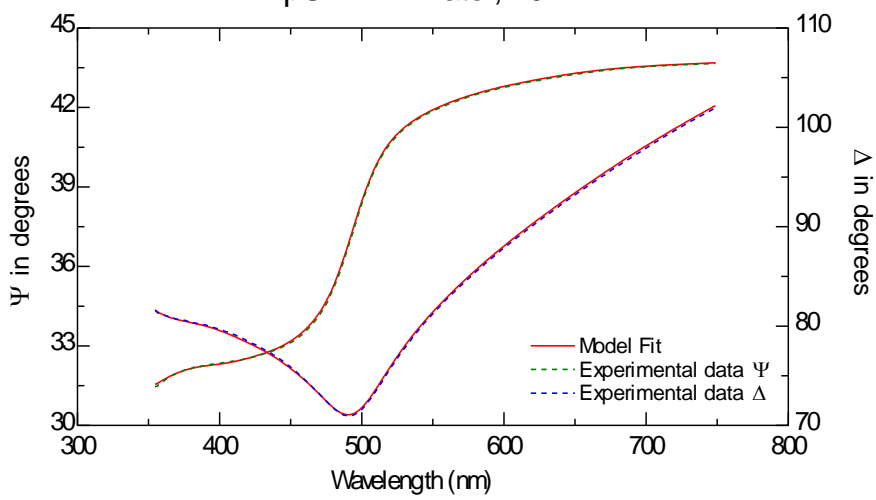

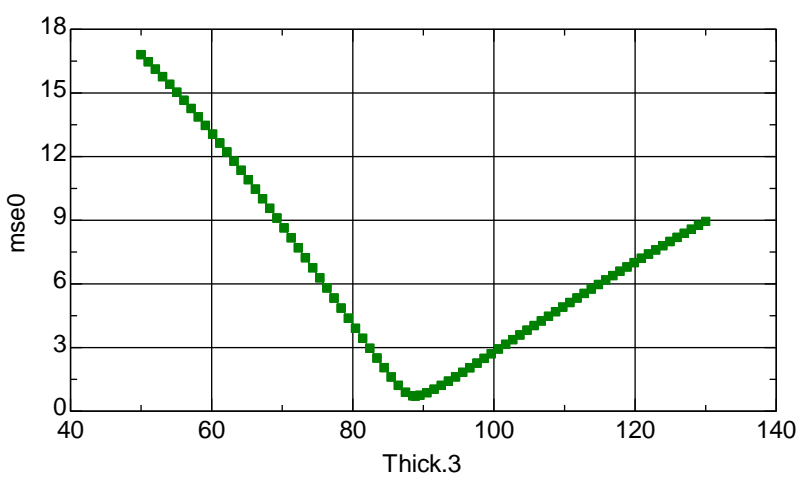

(b) Oscillators: $2 x$ Gaussian (amplitude1 $24.1 \pm 132$, En1 $99.88 \pm 514$, Br1 $5.04 \pm 2.98$; amplitude2 $0.10 \pm 0.09$, En2 8.38 $\pm 2.09, \mathrm{Br} 25.93 \pm 1.17$ ), MSE 0.78.
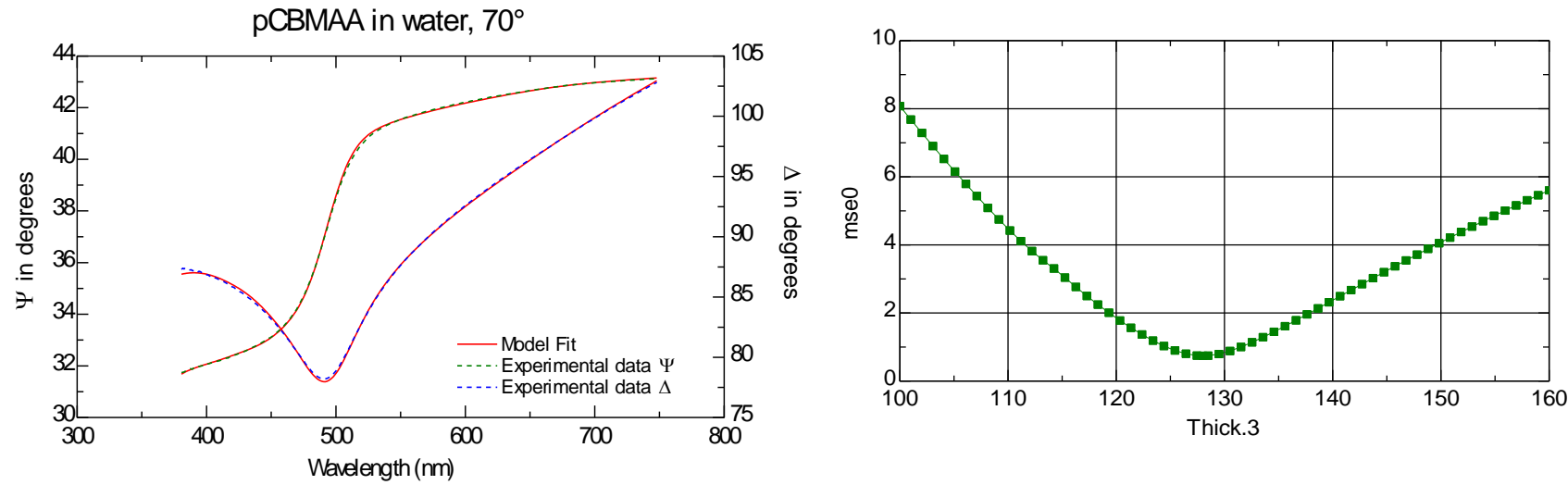

(c) Oscillators: 1x Tauc-Lorentz (amplitude $1.40 \pm 0.09$, Eg $1.51 \pm 0.10$, C $1.36 \pm 0.16$ ), MSE 0.74.
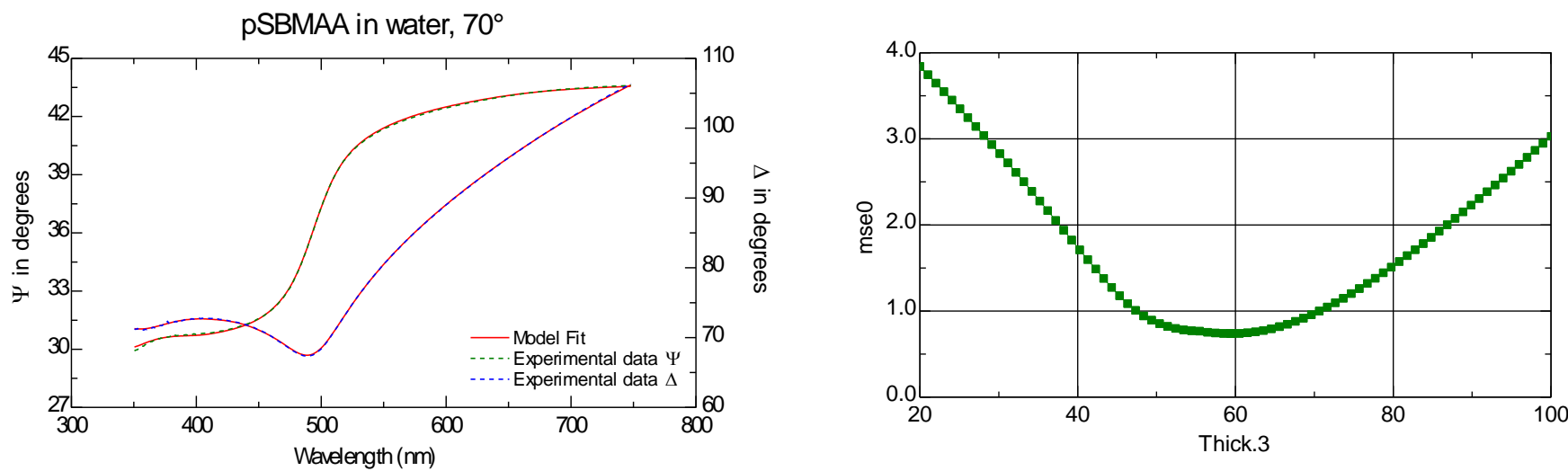
(d) Oscillators: $2 x$ Gaussian (amplitude1 $0.010 \pm 0.004$, En1 $3.02 \pm 0.14, \operatorname{Br} 10.93 \pm 0.29$; amplitude2 $0.83 \pm 0.53$, En2 $8.49 \pm 7.14, \mathrm{Br} 23.28 \pm 6.03)$, MSE 0.56.
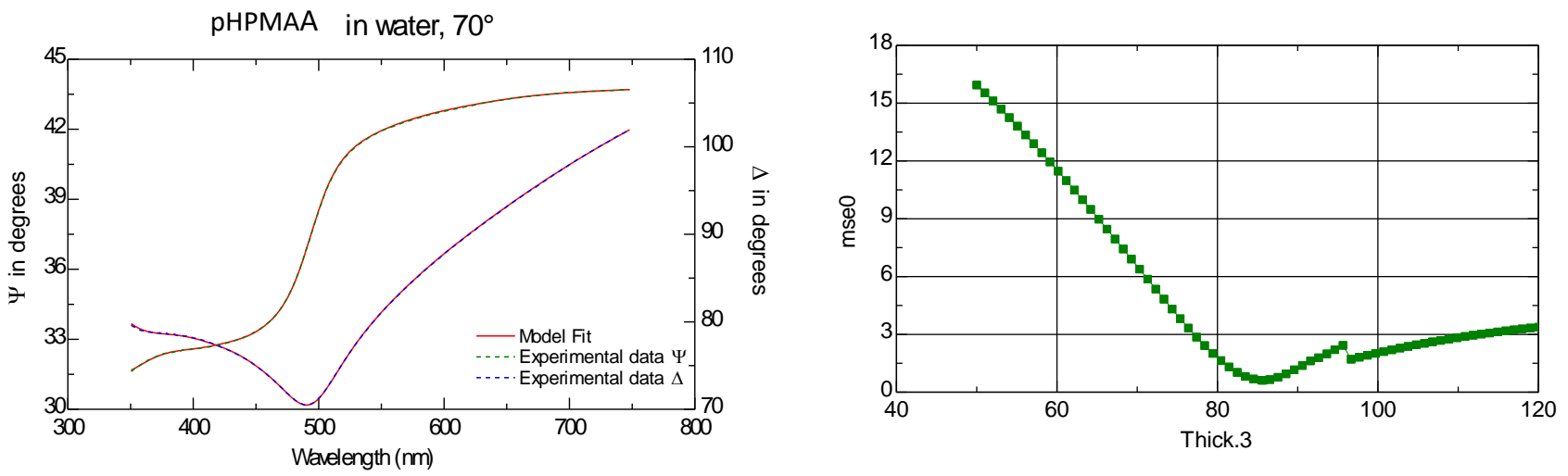

Figure S3. Representative experimental ellipsometric data taken from each polymer brush structure, along with model fits: (a) pCBAA, (b) pCBMAA, (c) pSBMAA, and (d) pHPMAA. Details on the oscillator model type, and used parameters (with errors), are given above for each brush type. The graphs on the right display the fit of thickness uniqueness.

\section{Gradient refractive index of pCBAA polymer brush with thickness of $242 \mathrm{~nm}$}
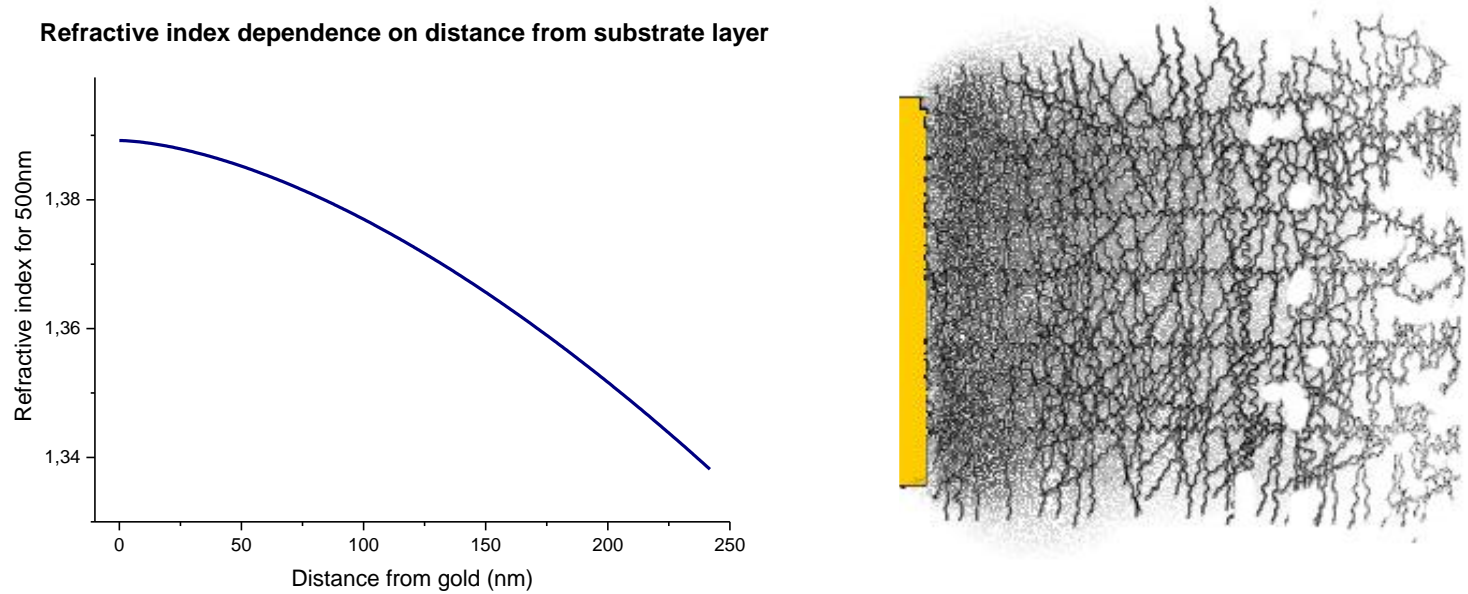

Figure S4: Left: Gradient refractive index (500 nm wavelength) measured from a pCBAA polymer brush in water (brush thickness $242 \mathrm{~nm}$ ). Right: Scheme of polymer brush with gradient density of polymer chains. 


\section{Infrared spectra of polymer brushes}

The IRRAS spectra of dry polymer brushes on gold were scanned using Thermo Scientific Nicolet iS50 FTIR Spectrometer equipped with the SAGA IRRAS module. The spectra were collected with 256 scans at $4 \mathrm{~cm}^{-1}$ resolution and $83^{\circ}$ reflection angle.

All the spectra show characteristic spectral bands of functional groups that confirm the structure of the polymers. The Amide I and II bands of the methacrylamide brushes (pHPMAA, pCBMAA, and pSBMAA) appear in the narrow range of $1650 \mathrm{~cm}^{-1}$ to $1654 \mathrm{~cm}^{-1}$ and $1529 \mathrm{~cm}^{-1}$ to $1533 \mathrm{~cm}^{-1}$, respectively. The absorptions of the acrylamide brush (pCBAA) occur at a bit higher frequencies, $1664 \mathrm{~cm}^{-1}$ and $1555 \mathrm{~cm}^{-1}$, respectively. Both carboxybetaines (pCBAA and pCBMAA) show intensive bands of ionized carboxyl group at $1612 \mathrm{~cm}^{-1}$ and $1363 \mathrm{~cm}^{-1}$, and also a rather weak diffusive band of non-ionized carboxyl groups at around $1725 \mathrm{~cm}^{-1}$. An extremely weak absorption in this region is also detected in the spectra of pHPMAA and PSBMAA, indicating the presence of some residual carboxyl groups. The sulfo group of PSBMAA is manifested in its spectrum by very intensive bands at $1212 \mathrm{~cm}^{-1}$ and $1040 \mathrm{~cm}^{-1}$ (asymmetric and symmetric stretching, respectively). ${ }^{1}$

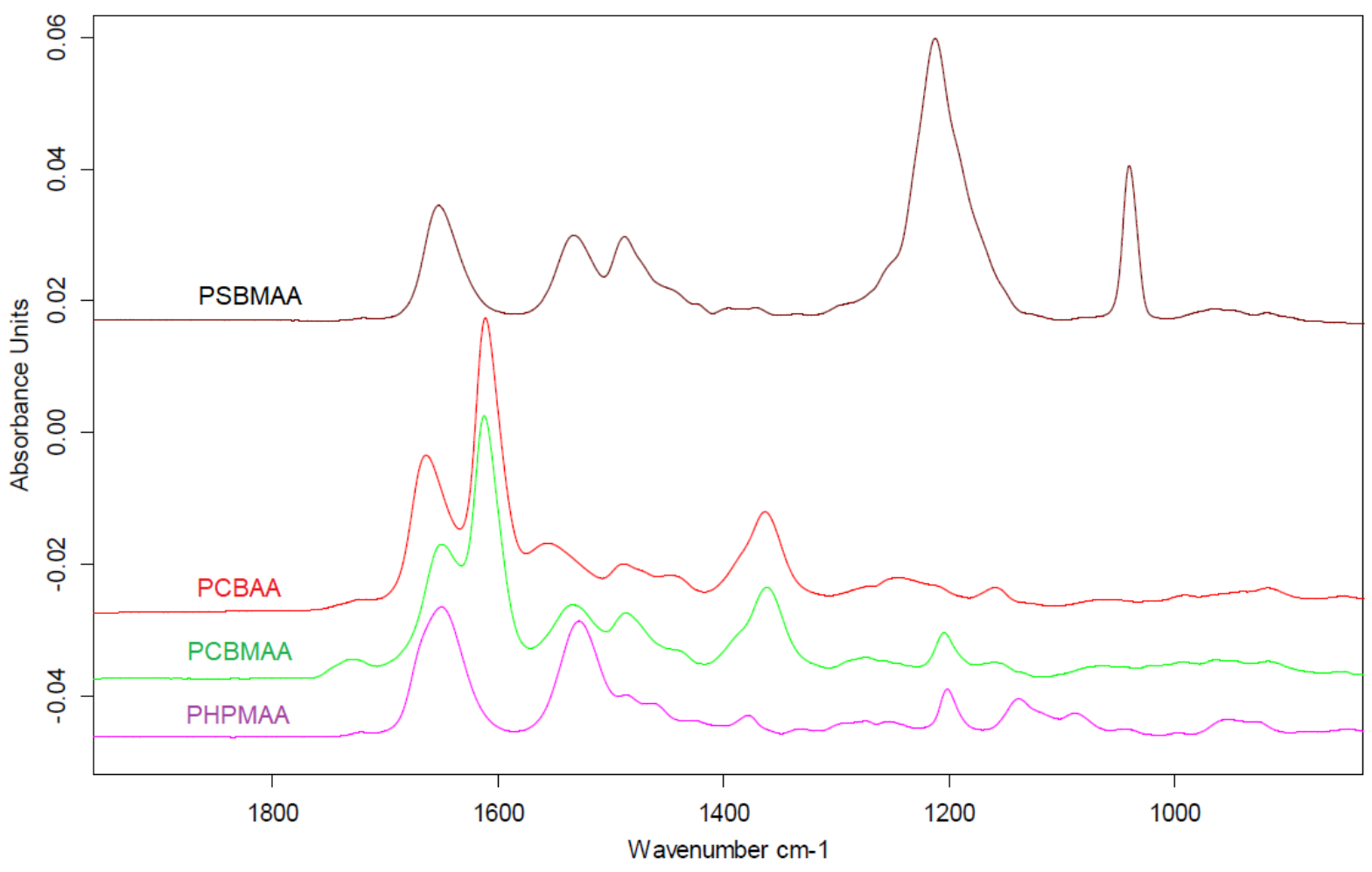

Figure S5: IRRAS spectra on the gold of the four polymer brushes. 


\section{Influence of different ionic strength of environment on swelling of polymer brushes: refractive index spectra}

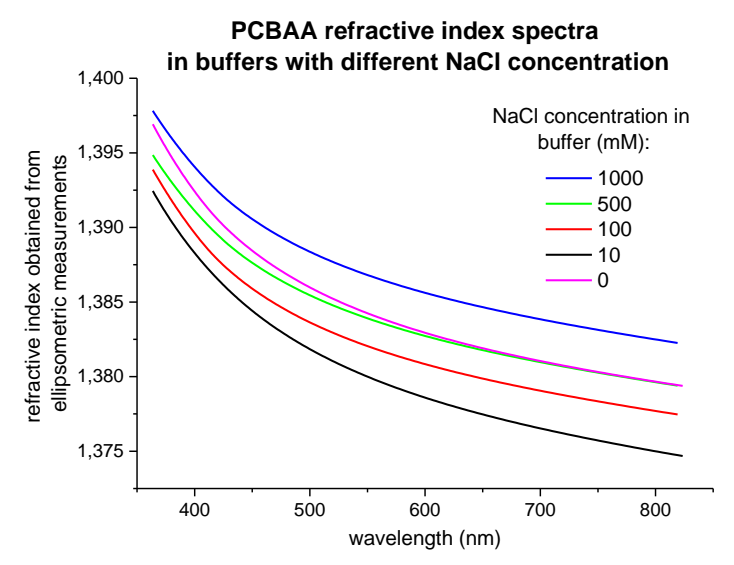

PCBMAA refractive index spectra

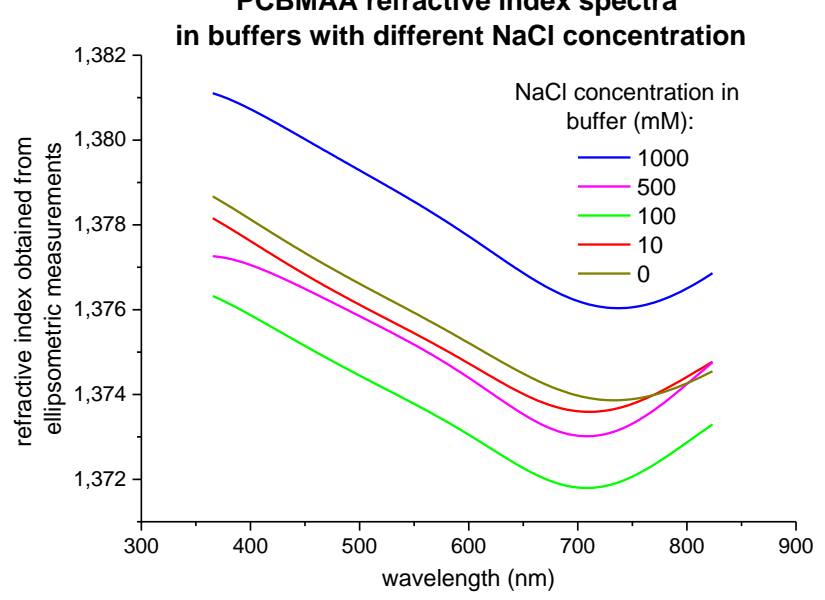

PSBMAA refractive index spectra in buffers with different $\mathrm{NaCl}$ concentration

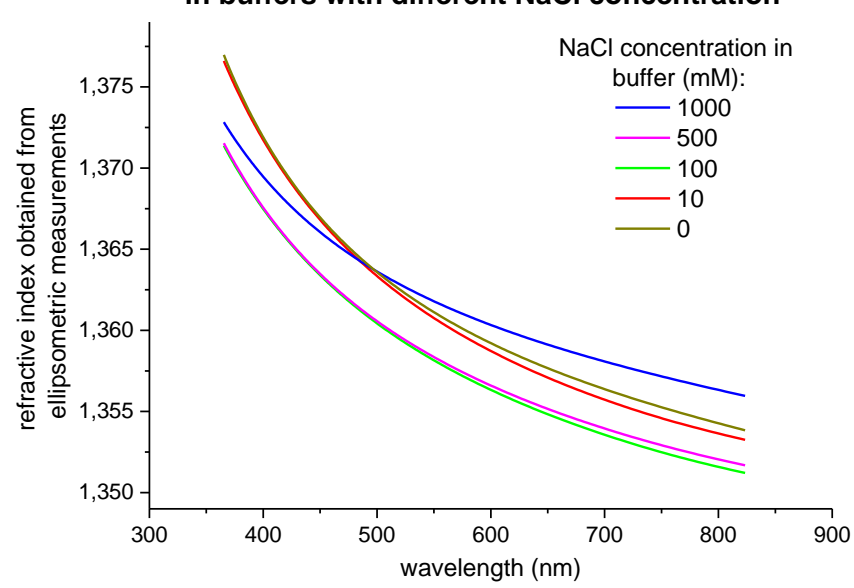

PCBAA refractive index spectra in buffers with different $\mathrm{MgCl}_{2}$ concentration

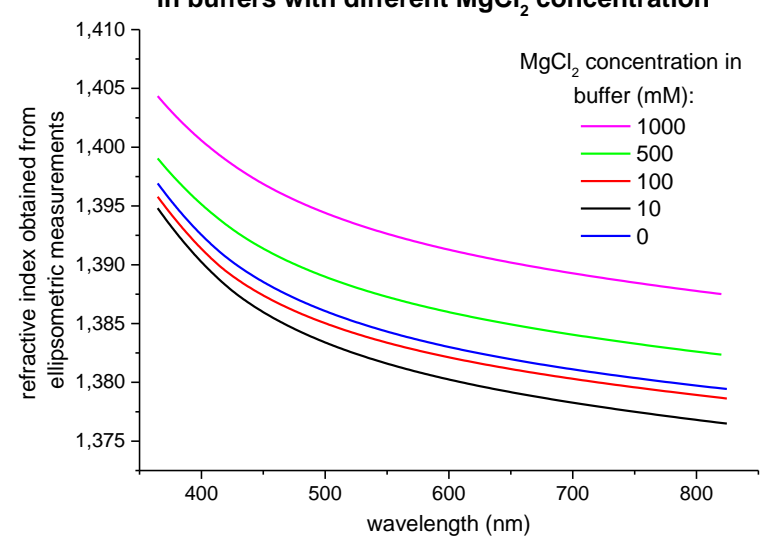

PCBMAA refractive index spectra in buffers with different $\mathrm{MgCl}_{2}$ concentration

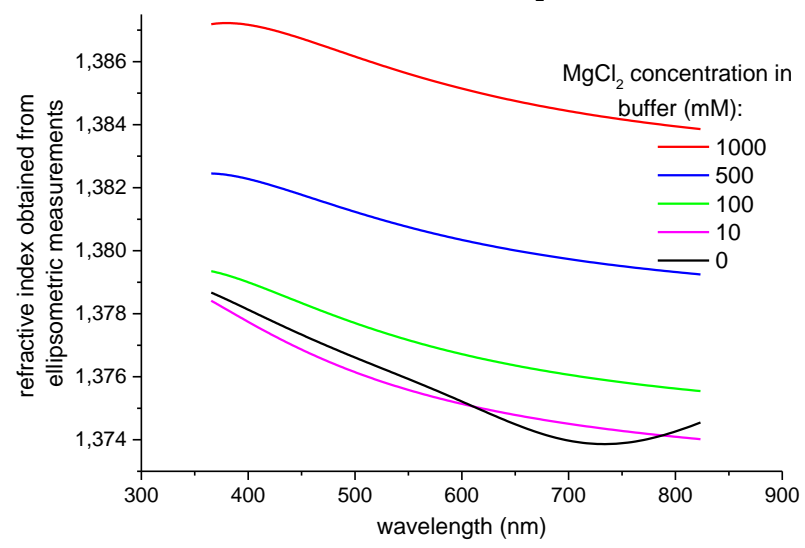

PSBMAA refractive index spectra in buffers with different $\mathrm{MgCl}_{2}$ concentration

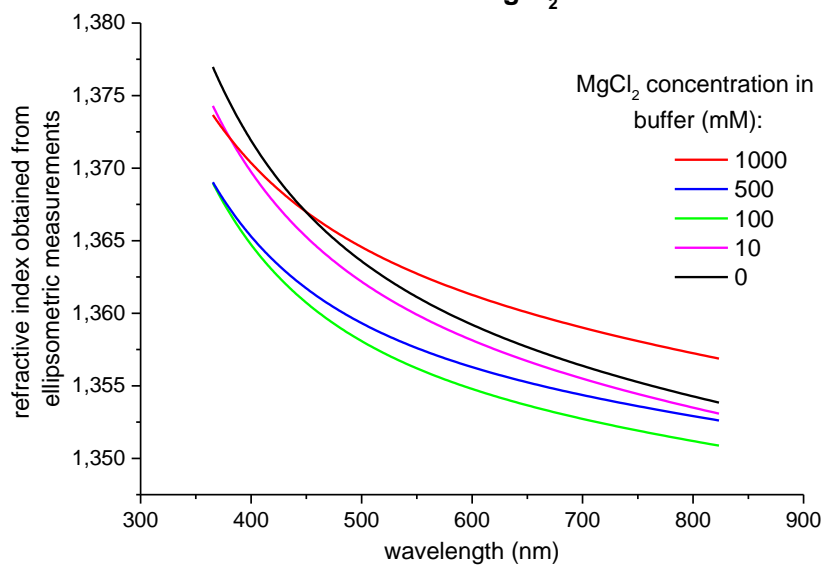


PHPMAA refractive index spectra in buffers with different $\mathrm{NaCl}$ concentration

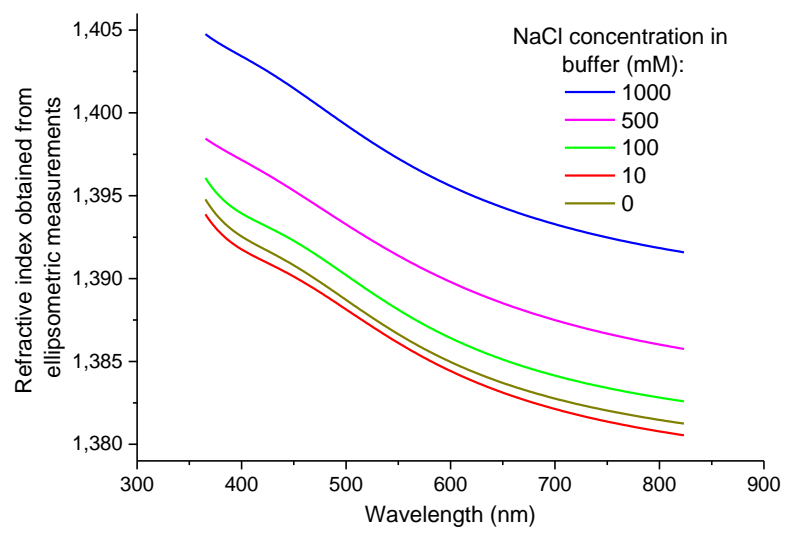

PHPMAA refractive index spectra in buffers with different $\mathrm{MgCl}_{2}$ concentration

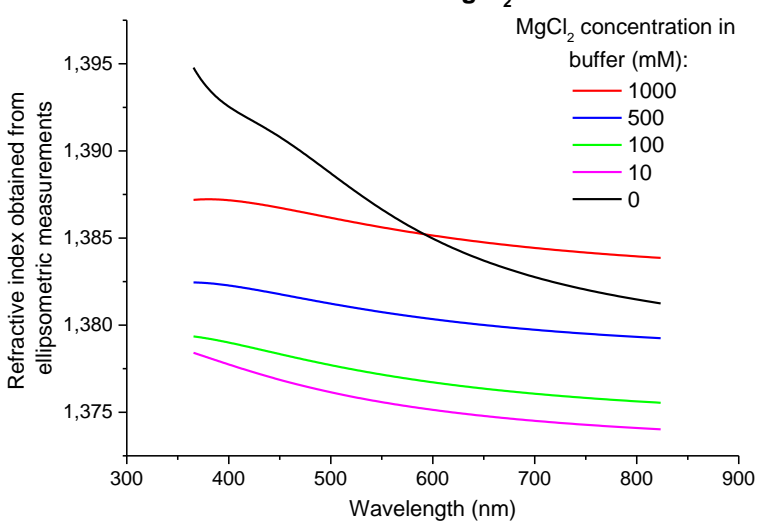

Figure S6: Refractive index spectra for all polymer brushes in different ionic strength environments for monovalent and bivalent chloride salts.

\section{Refractive index change after rehydration}

Refractive index spectra of polymer layers befor drying and after rehydration

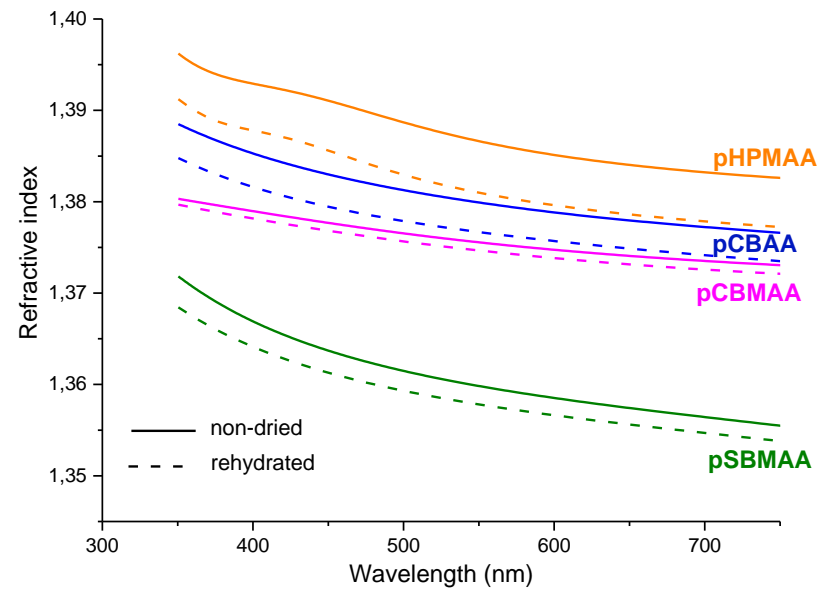

Figure S7: Refractive index spectra of polymer wet non-dried brush and wet rehydrated brush layers. 


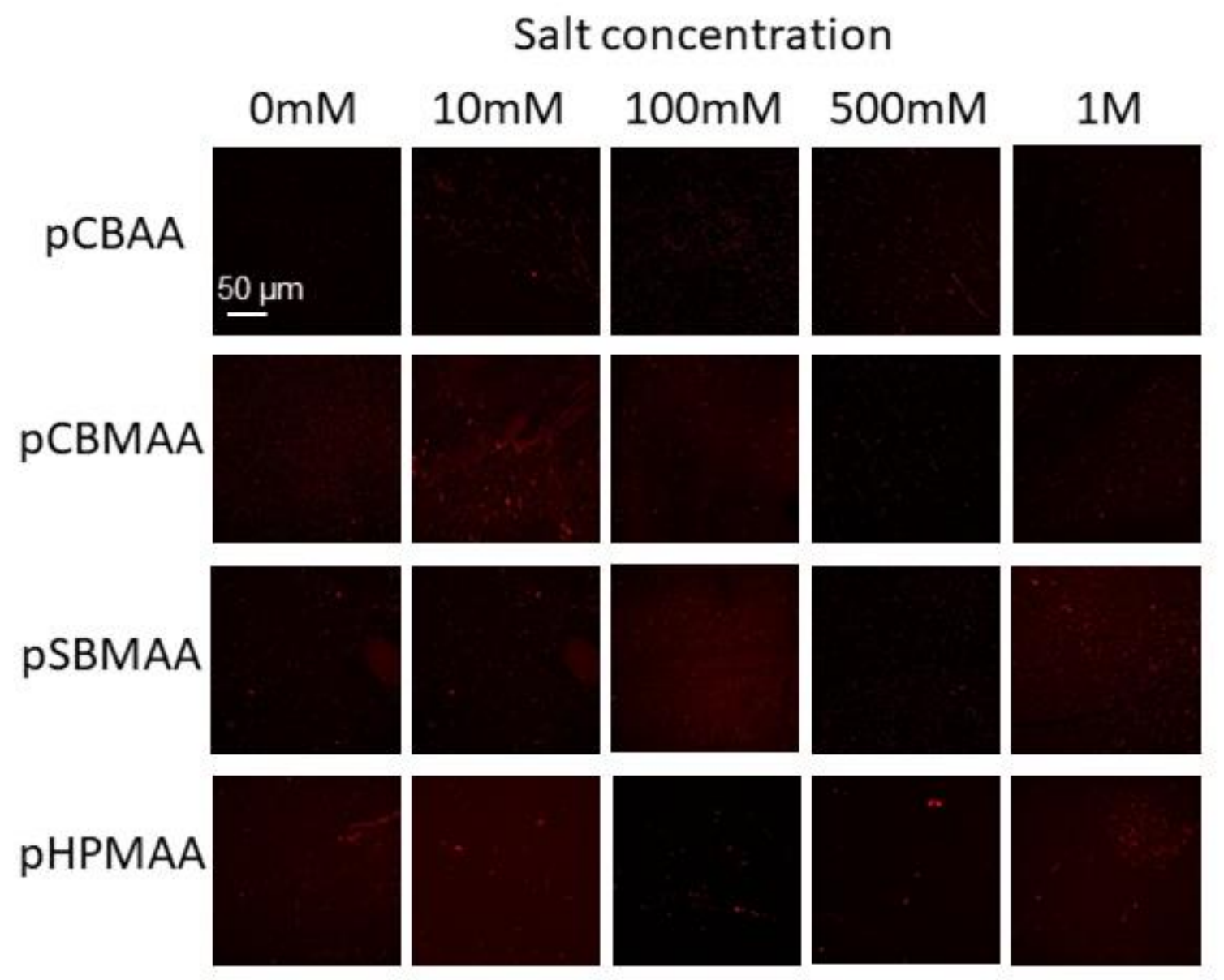

Figure S8: Representative pictures from fluorescent microscopy measurements: the fouling resistance of different polymer brushes to undiluted human blood plasma. Before the injection of plasma, coatings were incubated for 20 min in buffers with different $\mathrm{NaCl}$ concentrations. 

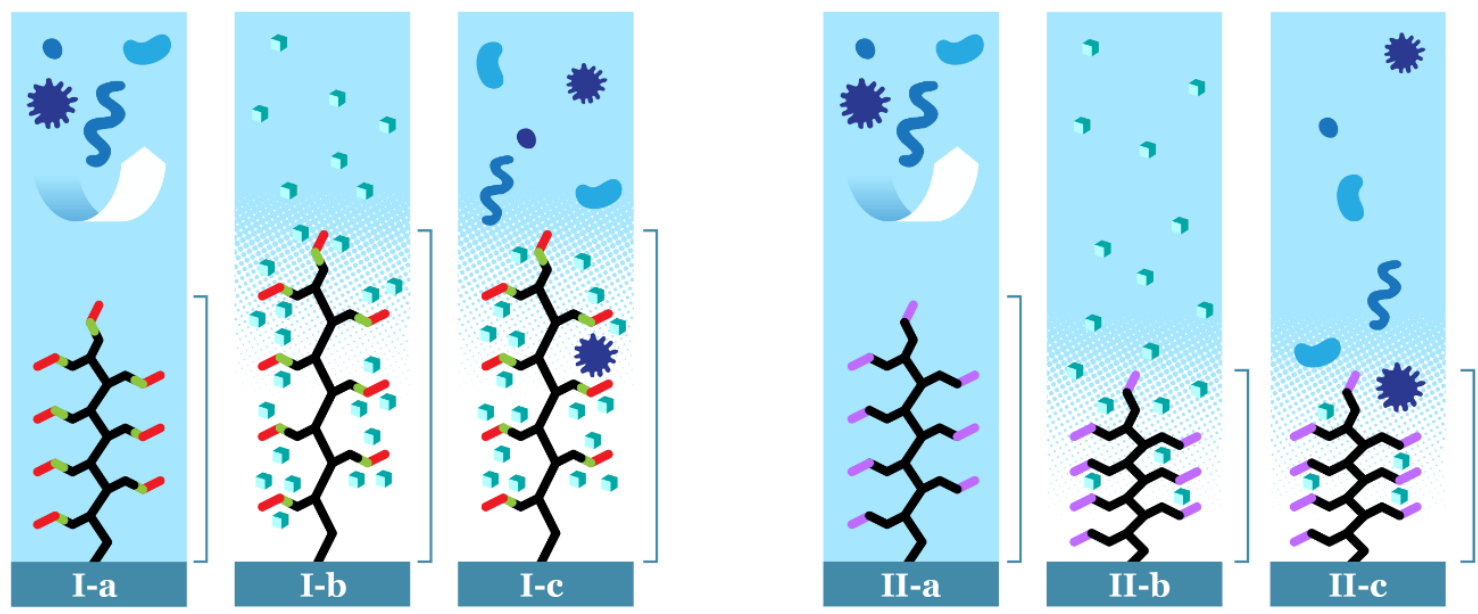

- coo-

NH3+

Salt ions

Biological medium

Figure S9: Scheme of the behavior of antifouling zwitterionic (I) and non-ionic (II) polymer brushes in the environment with different salt ions concentrations. After exposure to a higher ionic strength environment, the thickness and refractive index of zwitterionic brushes grow, while non-ionic brush shrinks (I-b and II-b). Such structural changes affect (positively or negatively) fouling from undiluted human blood plasma (I-C and II-C).

\section{References}

(1) Socrates, G. Infrared and Raman characteristic group frequencies : tables and charts, 3rd ed.; Chichester : Wiley, 2004. 